\title{
Frequency of meals and snacks are differentially associated with weight status in healthy weight and centrally obese 9-10-year-old children
}

\author{
A. Jennings ${ }^{1}$, A. Cassidy ${ }^{1}$, E. van Sluijs ${ }^{2}$, S. Griffin ${ }^{2}$ and A. Welch ${ }^{1}$ \\ ${ }^{1}$ Department of Nutrition, Norwich Medical School, University of East Anglia, Norwich NR4 7TJ, UK \\ and ${ }^{2}$ MRC Epidemiology Unit, Addenbrookes Hospital, Cambridge CB2 OQQ, UK
}

The rising prevalence of childhood obesity is a key public health issue worldwide. Eating frequency is one aspect of the diet that may be associated with weight status in children, although the mechanisms are unclear. We tested the hypothesis that increased eating frequency is associated with improved weight status in children, and differences in the distribution of eating occasions, the nutritional composition of the diet and physical activity levels would be observed between the most and least frequent eaters. Additionally, as overweight children have been shown to have different dietary and physical activity patterns compared with healthy weight children we examined whether the same relationships between eating frequency and weight status were observed in healthy-weight and centrally-obese children.

Cross-sectional data from 1700 children taking part in the SPEEDY study ${ }^{(1)}$ were analysed to examine associations between eating frequency, estimated from 4-d food diaries and objectively-measured weight status. Differences in distribution of eating occasions, meal composition and moderate and vigorous physical activity levels, measured by ActiGraph accelerometer, were then examined between the most- and least-frequent eaters. All associations were examined separately in healthy-weight and centrally-obese children. Child appropriate waist-to-height ratio cut-offs of 0.46 for boys and 0.45 girls were used to define central obesity ${ }^{(2)}$.

After multiple adjustments, including dietary under-reporting and physical activity, eating frequency was inversely associated with weight, BMI, BMI $z$ _score and waist circumference in healthy-weight children, and positively associated with BMI $z \_s c o r e$, and waist-toheight ratio in centrally obese children.

\begin{tabular}{|c|c|c|c|c|c|c|c|c|c|}
\hline & \multicolumn{3}{|c|}{ All children $(n 1700)$} & \multicolumn{3}{|c|}{ Healthy-weight children $(n$ 1034) } & \multicolumn{3}{|c|}{ Centrally-obese children $(n$ 666) } \\
\hline & B & SE & $P$ value & B & SE & $P$ value & B & $\mathrm{SE}$ & $P$ value \\
\hline Weight (kg) & -0.02 & 0.31 & 0.479 & -0.78 & 0.24 & 0.001 & 0.64 & 0.52 & 0.220 \\
\hline BMI $\left(\mathrm{kg} / \mathrm{m}^{2}\right)$ & 0.02 & 0.12 & 0.867 & -0.17 & 0.07 & 0.020 & 0.30 & 0.18 & 0.088 \\
\hline BMI (z_score) & -0.03 & 0.04 & 0.556 & -0.10 & 0.04 & 0.014 & 0.09 & 0.05 & 0.047 \\
\hline Waist circumference $(\mathrm{cm})$ & 0.41 & 0.31 & 0.893 & -0.38 & 0.17 & 0.031 & 0.71 & 0.45 & 0.118 \\
\hline Waist:height $(\mathrm{cm})$ & 0.002 & 0.002 & 0.313 & 0.000 & 0.001 & 0.849 & 0.005 & 0.003 & 0.036 \\
\hline Body fat $(\%)$ & -0.03 & 0.27 & 0.992 & -0.20 & 0.21 & 0.347 & 0.40 & 0.33 & 0.226 \\
\hline
\end{tabular}

Values are regression coefficients (B) and SE for the predicted difference in weight status per increase in one eating occasion; gender, parental education, under-reporting, energy intake and physical activity were included as covariates.

Compared with children in the lowest quartile of eating frequency, children who ate most frequently consumed breakfast more frequently (healthy weight $10 \% P<0.001$; centrally obese $9 \% P<0.001$ ) and breakfasts lower in fat (healthy weight and centrally obese $-18 \% P<0.001$ ). These children also consumed more snacks (healthy weight $188 \% P<0.001$; centrally obese $200 \% P<0.001$ ) and more fruit and vegetables from snacks (healthy weight $201 \%, P<0.001$; centrally obese $209 \% P<0.001$ ). Healthy-weight children who ate most frequently did significantly more vigorous activity than those who ate least frequently $(4 \%, P=0.033)$, unlike the centrally-obese children $(-2 \%, P=0.801)$

Increased eating frequency was associated with better dietary composition of breakfast and snacks in these 9-10-year-old UK children. In healthy-weight children, inverse associations between eating frequency and weight status were observed and those who ate most frequently reported significantly higher levels of vigorous physical activity. The underlying reason for the negative associations between eating frequency and weight status in centrally-obese children merits further investigation.

1. van Sluijs E, Skidmore P, Mwanza K et al. (2008) BMC Public Health 8, 388

2. Nambiar S, Hughes I \& Davies PS (2010) Public Health Nutr 13, 1566-1574. 